AperTO - Archivio Istituzionale Open Access dell'Università di Torino

Role of Invasive and Non-invasive Imaging Tools in the Diagnosis and Optimal Treatment of Patients with Spontaneous Coronary Artery Dissection

This is a pre print version of the following article:

Original Citation:

Availability:

This version is available http://hdl.handle.net/2318/1716064

since 2019-11-19T12:37:58Z

Published version:

DOI:10.1007/s11886-019-1202-0

Terms of use:

Open Access

Anyone can freely access the full text of works made available as "Open Access". Works made available under a Creative Commons license can be used according to the terms and conditions of said license. Use of all other works requires consent of the right holder (author or publisher) if not exempted from copyright protection by the applicable law. 


\section{Role of Invasive and Non-invasive Imaging Tools in the Diagnosis and Optimal Treatment of Patients with Spontaneous Coronary Artery Dissection.}

Enrico Cerrato1, Federico Giacobbe2, Cristina Rolfo1, Giorgio Quadri1, Francesco Tomassini1, Fabio Ferrari1, Fabio Mariani1, Matteo Anselmino2, Matteo Bianco3, Davide Belliggiano2, Luca Lo Savio4, Alfonso Franzé4, Umberto Barbero5, Italo Porto6, Hernán Mejía-Rentería7, Fernando Macaya7, Pablo Salinas8, Nieves Gonzalo7, Javier Escaned7, Laura Montagna3, Ferdinando Varbella1

\footnotetext{
1 Interventional Cardiology Unit, Orbassano, and Infermi Hospital, San Luigi Gonzaga University Hospital, Rivoli, Turin, Italy 2 Division of Cardiology, “Città della Salute e della Scienza di Torino” Hospital, Department of Medical Sciences, University of Turin, Turin, Italy 3 Department of Cardiology, San Luigi Gonzaga University Hospital, Orbassano, Turin, Italy 4 Cardiology Division, Infermi Hospital, Rivoli, Turin, Italy

5 Cardiology Division, Santissima Annunziata Hospital, Savigliano, Italy

6 Interventional Cardiology Unit, San Martino Hospital, University of Genoa, Genoa, Italy 7 Hospital Clinico San Carlos IDISCC, Complutense University of Madrid, Madrid, Spain 8 Servicio de Cardiologia, Hospital Clinico San Carlos, Instituto de Investigacion Sanitaria del Hospital Clinico San Carlos (IdISSC), Madrid, Spain
}

Word Count: 3048

Corresponding Author: Enrico Cerrato MD, Interventional Cardiology Unit, San Luigi Gonzaga University Hospital, Orbassano and Rivoli Infermi Hospital, Rivoli (Turin) Italy.

Email: enrico.cerrato@gmail.com, website: www.cardiogroup.org 


\section{ABSTRACT}

The latest advances in imaging technology have provided cardiologists with additional tools to help dealing challenging scenarios. A perfect example of such cases is represented by Spontaneous Coronary Artery Dissection (SCAD), which is often difficult to recognize and whose interventional treatment is not free from complications.

New techniques, such as IVUS, OCT or CCTA, have improved our ability to confirm doubtful cases of SCAD, optimize the interventional strategy and properly monitor patients after discharge. Hence, in the present review, we discuss the main imaging tools employed in SCAD cases, introducing their advantages and drawbacks and focusing on the different role they may play in SCAD diagnosis, management and follow-up

\section{INTRODUCTION}

Spontaneous coronary artery dissection (SCAD) is an uncommon cause of acute coronary syndrome (ACS) caused by the separation between the layers of the coronary artery wall. By definition, this must not be correlated to external trauma, coronary involvement of aortic dissection, iatrogenic causes nor complicated atherosclerosis ${ }^{1,2}$. This particular pathogenesis implies the development of an intramural hematoma (IMH) compressing the true lumen of the vessel: the new "false" lumen may rapidly expand reducing or even blocking the coronary flow, therefore leading to myocardial ischemia. Clinical presentation is often ACS but some cases present as ventricular arrhythmias or cardiac arrest.

SCAD has always been considered as a rare cause of myocardial infarction but increased awareness and encouragement in use of intracoronary imaging made clear in the latest studies that this is an underdiagnosed disorder, prevailing especially among young women without conventional cardiovascular risk factors (accounting for $15-35 \%$ of ACS in young women) $)^{1,3,4}$. 
Indeed, in the past this condition was barely diagnosed and classified but the widespread use of OCT and IVUS, in addition to the improved pathophysiological and clinical knowledge about the disease, has made it possible to increase the number of properly diagnosed cases, highlighting the urgent need to define the best diagnostic, therapeutic and follow-up pathways.

There are two proposed mechanisms for SCAD pathogenesis, which may present with different angiographic patterns. The first is the "inside-out" model, based on the development of an intimal tear while the second is the "outside-in" model, which implies a disruption of the coronary vasa vasorum.

Although the final result is always the intramural hematoma $(\mathrm{IMH})$, the angiographic appearance differs according to the pathophysiological cause ${ }^{5}$ : the inside-out mechanism often leads to the typical multiple radiolucent lumen, contrast dye stains in the arterial wall or even slow clearing or hang up of contrast dye is visible; the outside-in on the contrary may appear only as luminal narrowing due to the external compression: given the rarity of this finding, it may be misinterpreted as atherosclerotic disease. Sometimes instead other causes of misdiagnosis are mild and smooth-walled stenoses as well as the involvement of distal and small arteries ${ }^{5}$.

Imaging has a fundamental role in diagnosing and follow-up of SCAD. The spontaneous dissection must be suspected in all cases presenting with relevant clinical features such as young age, female gender, peri-partum, absence of conventional risk factors, etc. First assessment is always based on angiographic appearance, but only through imaging it is possible to eventually confirm the diagnosis and classify SCAD. There are SCAD variants that mimic atherosclerotic lesions or overlying spasms: for this reason, the current diagnostic algorithm for SCAD is based on different 
tools such as OCT and IVUS in addition to the coronary angiography that still represents the main diagnostic technique $e^{4,5}$.

Depending on the angiographic features three different types of SCAD were originally described ${ }^{2,6}$ :

- Type 1: Typical radiolucent "flap" with a double lumen image due to a linear filling defect, often associated with contrast hold-up. This pattern is easily distinguishable from catheter or interventional procedure-induced dissections where contrast readily enters the false lumen.

- Type 2: Long smooth stenosis which is mainly located in the mid or distal segments of the artery. It is divided in two subtypes:

o Type 2a: the distal vessel maintains a normal calibre

o Type $2 \mathrm{~b}$ : the stenosis angiographically reaches the distal tip of the vessel

- Type 3: Angiographically indistinguishable from focal or tubular atherosclerotic stenosis and requiring OCT or IVUS to demonstrate the presence of IMH and/or double lumen

The 2018 ESC position paper has introduced an additional Type $4^{1}$ which is described as the total occlusion of a vessel (usually a distal one) where sources of coronary embolism have been excluded: for this type there is supplementary evidence of complete vessel healing in keeping with the natural history of SCAD.

Type 2 and 3 are the most challenging to differentiate from atherosclerosis: the most common SCAD features are lack of atherosclerosis in other territories, long lesions and linear stenosis. Even though the clinical and angiographical suspicion is fundamental, the only way to immediately confirm diagnosis is through an intracoronary imaging device. Less frequently, a provisional 
diagnosis is made and the patient is scheduled for a control angiography when a definite diagnosis is made after healing of the affected segment.

Notably, any proposed flow-chart for SCAD treatment implies to perform, as a first step, a coronary angiogram. However, in the setting of SCAD, beside the diseased segment, all the coronary tree is more prone to develop other iatrogenic dissections even when catheters are carefully manipulated. For this reason, among intracoronary imaging, a potential role of noninvasive techniques such as cardiac tomography was also investigated by several authors. The cardiac computed tomography angiography (CCTA) is acquiring more and more importance as an efficient diagnostic tool in low and intermediate-risk patients with ACS. Actually, the computed tomography may assess coronary wall thickness and plaque constitution in addition to the presence of a double lumen ${ }^{7}$. Moreover, it is a non-invasive tool which permits to avoid the risk of iatrogenic dissection often reported with angiography.

Unfortunately, owing to its low diagnostic accuracy especially for the smaller tracts, CCTA must not be recommended as first-line exam to rule out $\mathrm{SCAD}^{1,5,8}$ : first of all it has a lower spatial resolution than the coronary angiography and SCAD usually affects the mid-to-distal tracts that are not well depicted by CCTA. Also, lumen compression by IMH and contrast staining may not be well visualized even with this non-invasive imaging tool ${ }^{9}$. The role of the computed tomography is to date limited to the follow-up assessment of SCAD, as presented in figure 1 . As invasive imaging techniques have been associated with a higher rate of iatrogenic complications the non-invasive tools are preferred for follow-up. Many studies about computed tomography coronary angiography have recently demonstrated CT may have greater utility in assessing healing where the site of dissection has already been determined by angiography. Furthermore, a Spanish prospective register conducted by Roura et al. has actually pointed out CCTA benefits in showing 
the vessel wall healing especially for those patients who did not undergo $\mathrm{PCl}^{10}$. MR angiography represents a radiation free alternative to CCTA for those patients presenting with renal insufficiency, diabetes or iodine-contrast intolerance ${ }^{11}$.

The use of Cardiac Magnetic Resonance (CMR) in SCAD patients has to date only been anecdotally described. It may be useful to confirm myocardial infarction and assess the extent of myocardial involvement but also to evaluate for other concurrent etiologies and sequelae ${ }^{12,13}$. Future investigations to elucidate the prognostic value of CMR parameters after acute SCAD are warranted.

Therefore, in the current review we will focus on the invasive imaging techniques, moving from diagnostic process to $\mathrm{PCl}$ planning/optimization and follow-up.

\section{INVASIVE IMAGING TECHNIQUES}

\section{CORONARY ANGIOGRAPHY}

In spite of being a bi-dimensional imaging tool not capable of imaging the arterial walls, coronary angiography represents today the first opportunity to diagnose a SCAD thanks to the typical angiographic and clinical features. Besides, as mentioned before, SCAD is currently classified with angiographic criteria.

Nevertheless, this exam always implies a greater risk for adding iatrogenic dissection in SCAD patients, because of the intrinsic frailty of the coronary walls ${ }^{14}$ and because the injection of contrast at high flow may cause SCAD progression. 


\section{INTRAVASCULAR IMAGING TECHNIQUES}

The intravascular imaging is being used for many purposes due to the possibility of showing the structure of the arterial wall and subsequently of the coronary plaques. These tools have led to greater accuracy but even to additional costs and complications ${ }^{3,15}$ : their advantages and limits are summarized in Suppl Figure A.

The firsts and main two main techniques are the intravascular ultrasound (IVUS) and the optical coherence tomography (OCT). Unfortunately, these new modalities imply further costs which prevent the intracoronary imaging from being always available. Moreover, both IVUS and OCT represent an invasive tool with potential risks, such as extending the dissection with the wire, the imaging catheter or the contrast injection (the latter for OCT only), catheter-induced iatrogenic dissections and catheter-induced occlusion of the true lumen ${ }^{2,3,16}$. The time from symptoms onset to intracoronary imaging could have an inverse relationship with complications ${ }^{15}$.

Thus, these investigations are usually recommended only for those uncertain cases which require a confirmatory diagnosis, as shown in Figure 2.

IVUS has a lower spatial resolution but a deeper penetration than OCT (4-8 rather than 1-2 mm) enabling the visualization of the entire vessel wall and an accurate measure of the depth and extent of the $\mathrm{IMH}^{3,8}$, not always available with $\mathrm{OCT}^{5}$. Unfortunately, it is worse than OCT in detecting intimal disruptions.

OCT, on the other hand, is a more recent tool in the clinical setting, whose benefits include improved ability to detect intimal disruptions, intraluminal clots, false lumens and IMH but requires blood clearance and has a limited optical penetration and shadowing ${ }^{1,2}$. Although most 
operators would prefer OCT for SCAD imaging, local experience with each of the intracoronary imaging tools and safety of additional contrast injections should be weighed to decide between OCT or IVUS.

Intravascular imaging has additional modalities that may be useful in order to better depict the false lumen and the intramural hematoma. In particular, ChromaFlo is an IVUS modality capable of identifying true and false lumen with a color interpolation: it compares sequential IVUS images and represents the two lumens with different colours by exploiting the echogenicity of blood particles $^{17}$.

Co-registration of OCT or IVUS with angiography is another modality often applied in order to better identify the vessel anatomy. This is able to assess the catheter position along the vessel with a series of frames acquired at the same time as the OCT or IVUS pullback. This allows a precise comparation of the angiography with the correspondent intravascular imaging frame helping in identifying specific patterns related to SCAD, as depicted in Figure 2.

\section{DIAGNOSTIC PROCESS}

Although the recognition of typical angiographic features and a more standardized use of intracoronary imaging is improving diagnostic accuracy, this condition remains under-recognized at the moment of diagnostic angiography ${ }^{6}$. Nevertheless, the potential risk of instrumenting an acutely dissected vessel should be carefully weighed, especially when the angiogram is characteristic of SCAD. 
As mentioned before, for those ambiguous cases of SCAD such as type 2 and 3 a supplementary tool is required. The type 2 variants are associated with very long lengths of narrowing and could be easily identified only after familiarity with this pattern, thereby OCT/IVUS or another angiography after 4-6 weeks should be considered in case of uncertainty (Figure 3). There is a higher risk of misdiagnosis for the type 3 because it may be mistaken for atherosclerosis unless intracoronary imaging is performed. This diagnostic difficulty was first noted by Maehara et coll. whose study showed 5 patients with IVUS proven SCAD had a medial dissection with an intramural hematoma occupying the dissected false lumen but none had intimal tears ${ }^{18}$. More recently these findings were confirmed by Alfonso et coll. who focused on the role of $\mathrm{OCT}^{19}$ : among 11 patients with confirmed SCAD, only three patients presented a classical angiographic intimal flap although a relatively diffuse lumen compromise was detected in all cases. In addition, mild stenosis, smooth walled stenosis and involvement of distal and small artery have also been reported in misdiagnosis by angiography of SCAD ${ }^{5}$. Taruya et al recently demonstrated that the recognizion by OCT of high risk features may help in stratifying patients with a poorer outcome ${ }^{20}$.

An example of diagnostic algorithm to be used for SCAD highlighting the role of intracoronary tools is presented in Suppl Figure B.

OCT may provide clearer images of the dissection site than IVUS and, when available, is preferred for diagnostic purposes owing to its better capability of visualizing the typical SCAD morphologic features (such as intimal flap and entry tear, double lumen morphology, intramural hematoma or associated thrombus) ${ }^{21}$.

Problems arise when the SCAD is distally located (especially type $2 b$ ). The inadequate display room for OCT in these cases could prevent the imaging of the entire affected segment or even the use of 
the tool at all ${ }^{6}$ (Suppl Figure C). Furthermore, when the vessel is large or the intramural hematoma is rich in red thrombi, the posterior structure cannot be seen clearly through $\mathrm{OCT}^{22}$, so IVUS may provide real-time imaging and the ChromaFlo modality may identify the site where the true and false lumen are connected.

Some authors suggest a combined use of both the intravascular techniques ${ }^{23}$ because they may provide unique diagnostic details on SCAD pathophysiological mechanism: this may be particularly relevant for those SCAD presenting with a different type on the follow-up. These studies have demonstrated both the tools have the same sensitivity in detecting the IMH but OCT still boasts an higher diagnostic accuracy for SCAD, in particular due to its better ability to recognize intimal ruptures and flaps ${ }^{23}$. Obviously, combination of both techniques implies to further prolong the procedure with higher procedural costs and risks.

\section{PCI PLANNING AND OPTIMIZATION}

An interventional management is currently discouraged as first line due to better outcomes of medically-treated patients (with beta-blockers and antiplatelet therapy above all) ${ }^{24}$ in addition to the potential iatrogenic damages with the guidewire which could lead to a subsequent interventional approach ${ }^{25}$ and to the typical distal localization that prevents stenting. Moreover, stenting itself may be followed by the "squeezing" of the intramural hematoma and increase of the risk of restenosis or stent thrombosis ${ }^{26}$. Thus, it is easy to understand why revascularization is to date advised only for those patients presenting with risk features such as STEMI presentation, hemodynamic instability, ventricular tachycardia or fibrillation, ongoing or recurrent episodes of angina or left main dissection ${ }^{27}$ and when surgery is not feasible. 
Even during $\mathrm{PCl}$, imaging obviously plays a fundamental role in order to avoid complications and optimize the interventional strategy. Both IVUS and OCT may be used to assess the presence of the guidewire within the true lumen: one of the most dangerous complications could actually be related to the performance of $\mathrm{PCl}$ in the false lumen with fatal results.

Many studies focused on the role of OCT during PCl in spontaneous coronary artery dissection ${ }^{19,22,28}$ : in particular OCT gives unique insights on the extension of hematoma and on the vessel diameter which contribute to determine an appropriate therapeutic strategy. This could be potentially useful for example in case of extensive hematoma in order to consider the use of cutting balloon to vent the hematoma avoiding proximal or distal propagation of the dissection, as described in few reports $^{29}$. Finally, the hematoma resorption could lead to stent/scaffold malposition, which may be prevented through an intracoronary imaging staged study to optimize the previous $\mathrm{PCl}$.

An excellent example of intravascular tools' utility in PCl planning is shown in Suppl Figure D.

\section{FOLLOW-UP}

The latest studies on SCAD have highlighted the importance of the angiographic follow-up. Indeed, in the vast majority of SCAD patients selected for conservative therapy the dissection heals within months at least from an angiographic point of view and the clinical outcome of these patients is generally favorable 30,31 . Most of the cases stable during the acute phase healed completely at least after 26 days from the index event ${ }^{6}$. These results warrant the "conservative whenever possible" approach.

An angiographic follow-up may be used at first to confirm SCAD diagnosis after an initial conservative treatment. Besides, it represents a way to ensure long term result after extensive 
stenting/scaffolding and even to assess the healing of the vessels involved. In particular, the evaluation of SCAD-healing may be relevant for decision-making on the duration of the antiplatelet therapy or in symptomatic patients with an uncertain diagnosis exclude recurrent SCAD after the first event.

Even intracoronary imaging may be used for the follow-up: OCT is usually preferred to IVUS owing to its better spatial resolution and is recommended after $\mathrm{PCl}$ to assess for malapposed/uncovered struts before stopping the antiplatelet therapy².

In most cases a complete restitutio ad integrum occurs and may be appreciated only through $\mathrm{OCT}^{19}$, notwithstanding this tool must be reserved only for those clinical situations that require a strong confirmation as the procedural risks often outweigh the potential benefits.

The last but not the least, intracoronary imaging may be used during follow-up in case of implant of a bio-resorbable scaffold in order to assess the complete resorption of the device ${ }^{32}$, a promising technique that may help to take care of the SCAD without leaving permanent stents in young patients.

\section{EXTRACARDIAC ARTERIOPATHIES}

Most reports show a high prevalence in SCAD patients of the so-called "extra-coronary vascular abnormalities" (EVAs) arousing suspicion SCAD could be a located manifestation of a systemic vascular disorder ${ }^{3,4}$. The most commonly reported is fibromuscular dysplasia (FMD) but even coronary tortuosity, focal stenoses and intracranial aneurisms with all the associated risks warrant the need for a screening protocol for EVAs in case of SCAD. Furthermore, patients presenting with severe vascular tortuosity are usually affected by a higher risk of SCAD recurrence ${ }^{33}$. 
Saw et coll. suggest searching for these abnormalities during the index coronary angiography for the potential impact on patient management ${ }^{2}$ but the high risks related to additional invasive imaging, especially for unstable patients, prompt operators to evaluate them during the followup ${ }^{11,34}$. Additionally, Liang et coll. ${ }^{34}$ have presented a protocol to detect EVAs in SCAD patients with low-osmolar contrast agents and low radiation doses given that SCAD usually affects young women and may be associated with pregnancy.

In this sense, contrast-enhanced magnetic resonance angiography has recently been proposed as a possible alternative to CCTA and angiography for the screening of EVAs with optimal results ${ }^{35}$.

\section{CONCLUSIONS}

In conclusion, imaging deserves an essential role in SCAD diagnosis and management. If coronary angiography represents to date the first choice to recognize SCAD, it may be supported by intravascular tools in order to confirm diagnosis and better describe the type of dissection. Intracoronary techniques may support the interventional management by providing unique insights on IMH extension and morphology as well as confirming, in case of needed invasive treatment, the presence of the guidewire in the true lumen and stent/scaffold apposition. Finally, in the follow up, the imaging techniques (included the non-invasive options) help to assess the long term result in case of extensive stenting/scaffolding, provide informations about the spontaneous healing when it is not performed (with a particular mention to the new CTA scanners as a non-invasive method to reduce costs and risks for the patients) and to rule out extracardiac arteriopathy.

\section{Competing interest}


All authors have completed the Unified Competing Interest form at

www.icmje.org/coi disclosure.pdf (available on request from the corresponding author) and declare: no support from any organisation for the submitted work; no financial relationships with any organisations that might have an interest in the submitted work in the previous 3 years; no other relationships or activities that could appear to have influenced the submitted work."

\section{Copyright Statement}

The Corresponding Author has the right to grant on behalf of all authors and does grant on behalf of all authors, a worldwide licence to the Publishers and its licensees in perpetuity, in all forms, formats and media (whether known now or created in the future), to i) publish, reproduce, distribute, display and store the Contribution, ii) translate the Contribution into other languages, create adaptations, reprints, include within collections and create summaries, extracts and/or, abstracts of the Contribution, iii) create any other derivative work(s) based on the Contribution, iv) to exploit all subsidiary rights in the Contribution, $v$ ) the inclusion of electronic links from the Contribution to third party material where-ever it may be located; and, vi) licence any third party to do any or all of the above.

\section{BIBLIOGRAPHY}

1. Adlam, D. et al. European Society of Cardiology, acute cardiovascular care association, SCAD study group: a position paper on spontaneous coronary artery dissection. Eur Heart J doi:10.1093/eurheartj/ehy080

2. Saw, J., Mancini, G. B. J. \& Humphries, K. H. Contemporary Review on Spontaneous Coronary Artery Dissection. J. Am. Coll. Cardiol. 68, 297-312 (2016).

3. Hayes, S. N. et al. Spontaneous Coronary Artery Dissection: Current State of the Science: A Scientific Statement From the American Heart Association. Circulation CIR.0000000000000564 (2018). doi:10.1161/CIR.0000000000000564 
4. Macaya, F. et al. Spontaneous coronary artery dissection: contemporary aspects of diagnosis and patient management. Open Heart 5, e000884 (2018).

5. Saw, J. Coronary angiogram classification of spontaneous coronary artery dissection. Catheter Cardiovasc Interv 84, 1115-1122 (2014).

6. Al-Hussaini, A. \& Adlam, D. Spontaneous coronary artery dissection. Heart 103, 1043-1051 (2017).

7. Das Neves, B. C. et al. Evolutive recanalization of spontaneous coronary artery dissection: insights from a multimodality imaging approach. Circulation 129, 719-720 (2014).

8. Alfonso, F. et al. Spontaneous coronary artery dissection: novel insights on diagnosis and management. Cardiovasc Diagn Ther 5, 133-140 (2015).

9. Eleid, M. F. et al. Spontaneous coronary artery dissection: challenges of coronary computed tomography angiography. Eur Heart J Acute Cardiovasc Care 7, 609-613 (2018).

10. Roura, G. et al. Noninvasive Follow-Up of Patients With Spontaneous Coronary Artery Dissection With CT Angiography. 9, (2016).

11. Toggweiler, S. et al. Associated vascular lesions in patients with spontaneous coronary artery dissection. Swiss Med Wkly 142, w13538 (2012).

12. Tan, N. Y., Hayes, S. N., Young, P. M., Gulati, R. \& Tweet, M. S. Usefulness of Cardiac Magnetic Resonance Imaging in Patients With Acute Spontaneous Coronary Artery Dissection. The American Journal of Cardiology 122, 1624-1629 (2018).

13. Nakashima, T. et al. Detection of intramural hematoma and serial non-contrast T1weighted magnetic resonance imaging findings in a female patient with spontaneous coronary artery dissection. Circ. J. 77, 2844-2845 (2013).

14. Lebrun, S. \& Bond, R. M. Spontaneous coronary artery dissection (SCAD): The underdiagnosed cardiac condition that plagues women. Trends in Cardiovascular Medicine 28, 340-345 (2018).

15. Macaya, F. et al. Feasibility and safety of intracoronary imaging for diagnosing spontaneous coronary artery dissection. JACC Cardiovasc Imaging, (2018).

16. Prakash, R., Starovoytov, A., Heydari, M., Mancini, G. B. J. \& Saw, J. Catheter-Induced latrogenic Coronary Artery Dissection in Patients With Spontaneous Coronary Artery Dissection. JACC Cardiovasc Interv 9, 1851-1853 (2016).

17. Deftereos, S. et al. Role of grey-scale intravascular ultrasound and ChromaFlo in deciding on treatment approach for spontaneous coronary dissection in a young woman. Hellenic J Cardiol 52, 364-366 (2011).

18. Maehara, A. et al. Intravascular ultrasound assessment of spontaneous coronary artery dissection. Am. J. Cardiol. 89, 466-468 (2002).

19. Alfonso, F. et al. Diagnosis of spontaneous coronary artery dissection by optical coherence tomography. J. Am. Coll. Cardiol. 59, 1073-1079 (2012).

20. Taruya, A. et al. Lesion characteristics and prognosis of acute coronary syndrome without angiographically significant coronary artery stenosis. European Heart Journal - Cardiovascular Imaging (2019). doi:10.1093/ehjci/jez079

21. Satogami, K. et al. Successful stenting with optical frequency domain imaging guidance for spontaneous coronary artery dissection. JACC Cardiovasc Interv 8, e83-e85 (2015).

22. Alfonso, F., Paulo, M. \& Dutary, J. Endovascular imaging of angiographically invisible spontaneous coronary artery dissection. JACC Cardiovasc Interv 5, 452-453 (2012).

23. Paulo, M. et al. Combined use of OCT and IVUS in spontaneous coronary artery dissection. JACC Cardiovasc Imaging 6, 830-832 (2013).

24. Tweet, M. S. et al. Spontaneous coronary artery dissection: revascularization versus conservative therapy. Circ Cardiovasc Interv 7, 777-786 (2014). 
25. Quadri, G. et al. Importance of Close Surveillance of Patients With Conservatively Managed Spontaneous Coronary Artery Dissection. JACC Cardiovasc Interv 11, e87-e89 (2018).

26. Cerrato, E. et al. Acute Interventional Management of Spontaneous Coronary Artery Dissection: Case Series and Literature Review. International Cardiovascular Forum Journal 15, (2019).

27. Saw, J. Natural history of spontaneous coronary artery dissection: to stent or not to stent? Eurolntervention 14, 1353-1356 (2019).

28. Cockburn, J., Yan, W., Bhindi, R. \& Hansen, P. Spontaneous Coronary Artery Dissection Treated With Bioresorbable Vascular Scaffolds Guided by Optical Coherence Tomography. Canadian Journal of Cardiology 30, 1461.e1-1461.e3 (2014).

29. Ito, T., Shintani, Y., Ichihashi, T., Fujita, H. \& Ohte, N. Non-atherosclerotic spontaneous coronary artery dissection revascularized by intravascular ultrasonography-guided fenestration with cutting balloon angioplasty. Cardiovasc Interv Ther 32, 241-243 (2017).

30. Rogowski, S. et al. Spontaneous Coronary Artery Dissection: Angiographic Follow-Up and Long-Term Clinical Outcome in a Predominantly Medically Treated Population. Catheter Cardiovasc Interv 89, 59-68 (2017).

31. Hassan, S., Prakash, R., Starovoytov, A. \& Saw, J. Natural History of Spontaneous Coronary Artery Dissection With Spontaneous Angiographic Healing. JACC: Cardiovascular Interventions (2019). doi:10.1016/j.jcin.2018.12.011

32. Quadri, G., Cerrato, E., Rolfo, C. \& Varbella, F. Spontaneous coronary artery dissection treated with magnesium-made bioresorbable scaffold: 1-Year angiographic and optical coherence tomography follow-up. Catheter Cardiovasc Interv (2018). doi:10.1002/ccd.27971

33. Eleid, M. F. et al. Coronary artery tortuosity in spontaneous coronary artery dissection: angiographic characteristics and clinical implications. Circ Cardiovasc Interv 7, 656-662 (2014). 34. Liang, J. J. et al. A novel application of CT angiography to detect extracoronary vascular abnormalities in patients with spontaneous coronary artery dissection. J Cardiovasc Comput Tomogr 8, 189-197 (2014).

35. Macaya, F. et al. Screening of extra-coronary arteriopathy with magnetic resonance angiography in patients with spontaneous coronary artery dissection: a single-centre experience. Cardiovascular Diagnosis and Therapy 0, (2019). 


\section{FIGURE CAPTIONS}

FIGURE 1: 50 year's old woman presenting with ACS/NSTEMI. After a conservative strategy treatment, patient was re-admitted few days after the discharge. Angiography showed a proximal and distal propagation of the SCAD requiring an extensive treatment with an hybrid DES and BRS DESSOLVE. However residual dissection with hematoma persist in the mid-distal part of the circumflex (red arrows). (Upper panel). Clinical follow-up was free of recurrences. AngioTC followup was performed one year later showing an excellent result with hematoma reabsorption and healing of the mid-distal part of the circunflex. DESSOLVE BRS markers was visible by angioTC (red asterisk)

FIGURE 2: 50 year's old woman presenting with ACS/NSTEMI. Angiographic finding was unclear. Optical Coherence Tomography interrogation allowed to clear identify the position of the guidewire in the true lumen identifying the true lumen on the vessel in course of SCAD.

FIGURE 3: 51 years old woman presenting with ACS/NSTEMI. Angiographic finding suspected for type 2 SCAD was confirmed by intravascular ultrasoung imaging (IVUS)

\section{FIGURE 1}

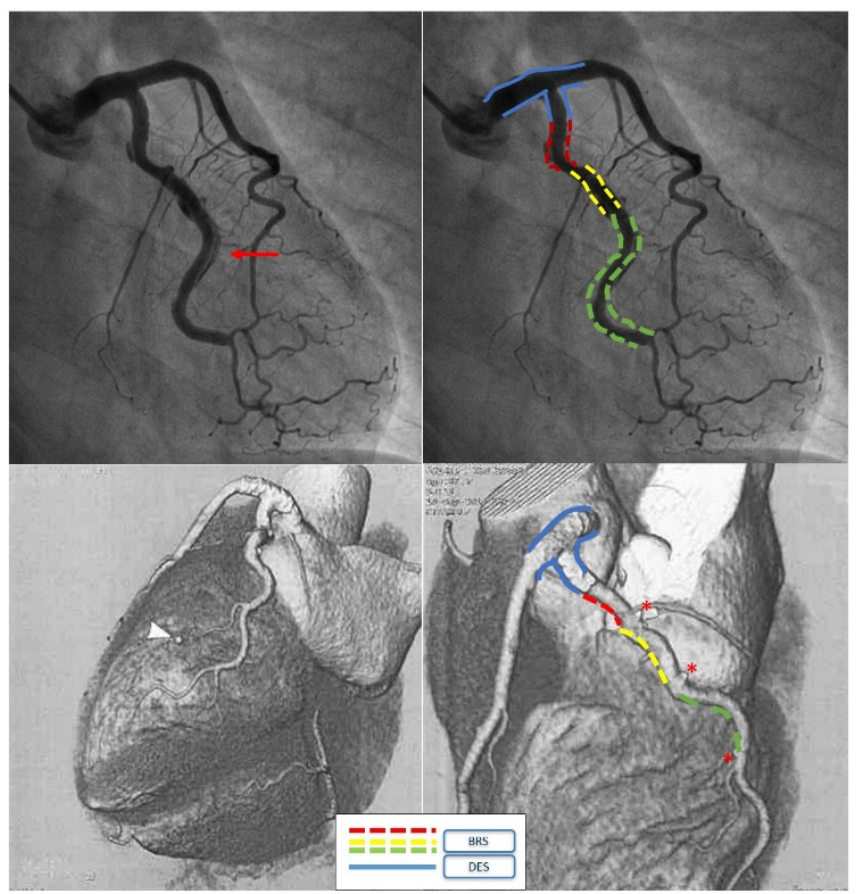


FIGURE 2

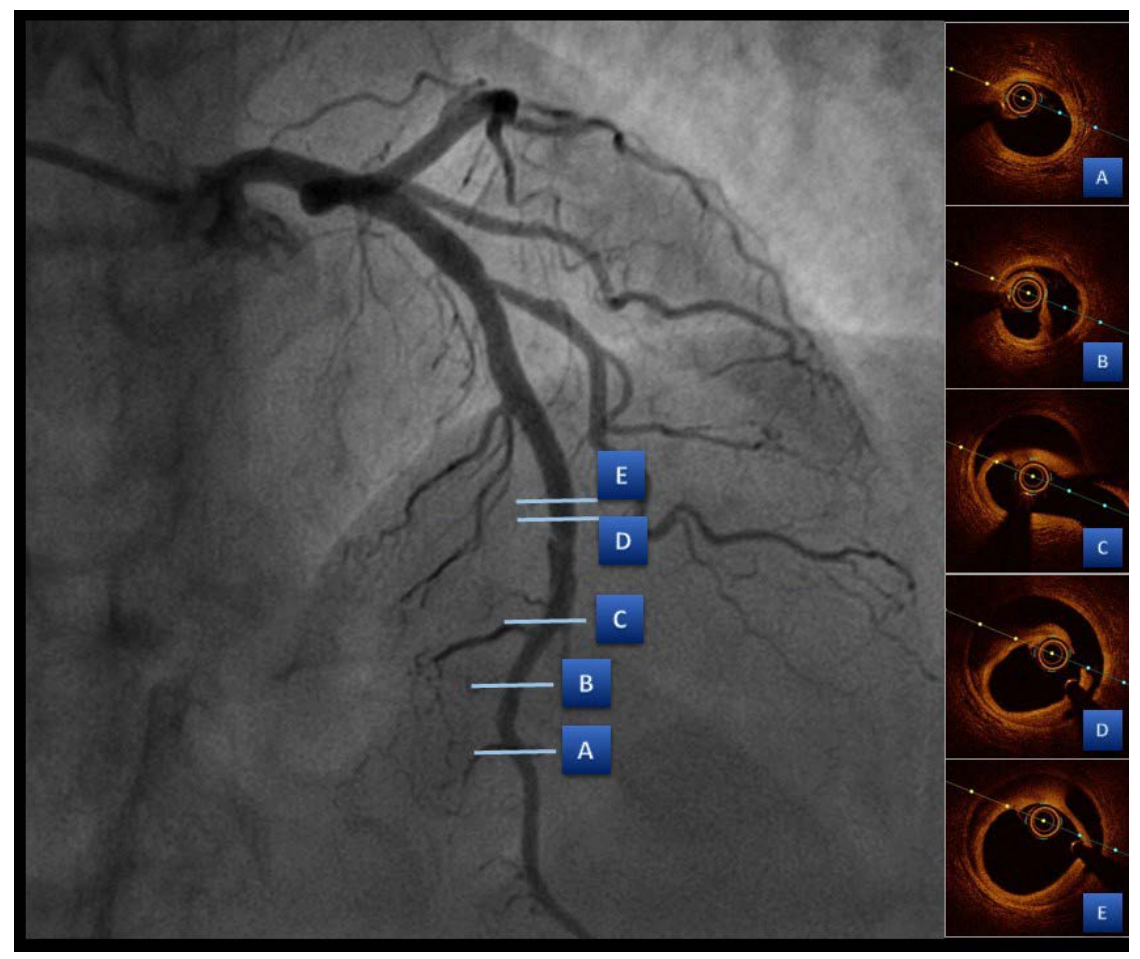

FIGURE 3

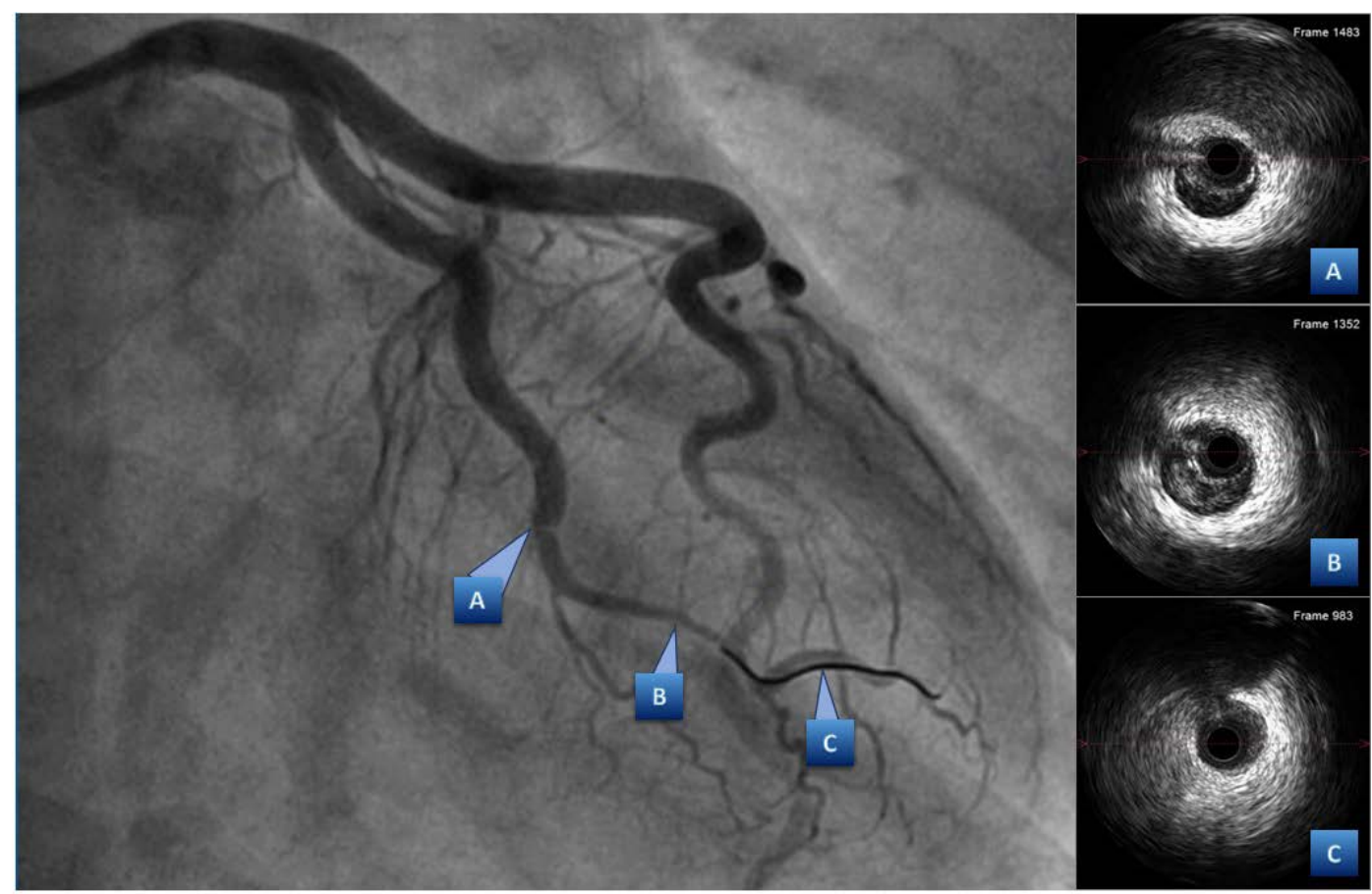

